\title{
Peripheral injection of human umbilical cord blood stimulates neurogenesis in the aged rat brain
}

\author{
Adam D Bachstetter1,2, Mibel M Pabon ${ }^{1,2}$, Michael J Cole ${ }^{2}$, \\ Charles E Hudson ${ }^{3}$, Paul R Sanberg1,2,4, Alison E Willing1,2, \\ Paula C Bickford ${ }^{1,2,3}$ and Carmelina Gemma*1,2,3
}

\begin{abstract}
Address: ${ }^{1}$ Department of Molecular Pharmacology and Physiology, University of South Florida, College of Medicine, Tampa, FL 33612, USA, 2Department of Neurosurgery, Center of Excellence for Aging and Brain Repair, University of South Florida, College of Medicine, Tampa, FL 33612, USA, ${ }^{3}$ James A. Haley Veterans Administration Hospital, Tampa, FL 33612, USA and ${ }^{4}$ Saneron CCEL Therapeutics Inc., Temple Terrace, FL 33617, USA

Email: Adam D Bachstetter - abachste@health.usf.edu; Mibel M Pabon - mpabon@health.usf.edu; Michael J Cole - mcole@health.usf.edu; Charles E Hudson - chudson1@ health.usf.edu; Paul R Sanberg - psanberg@health.usf.edu; Alison E Willing - awilling@health.usf.edu; Paula C Bickford - pbickfor@health.usf.edu; Carmelina Gemma* - cgemma@health.usf.edu

* Corresponding author
\end{abstract}

Published: 14 February 2008

BMC Neuroscience 2008, 9:22 doi:10.1 I86/147|-2202-9-22
Received: 3 January 2008

Accepted: 14 February 2008

This article is available from: http://www.biomedcentral.com/I47/-2202/9/22

(c) 2008 Bachstetter et al; licensee BioMed Central Ltd.

This is an Open Access article distributed under the terms of the Creative Commons Attribution License (http://creativecommons.org/licenses/by/2.0), which permits unrestricted use, distribution, and reproduction in any medium, provided the original work is properly cited.

\begin{abstract}
Background: Neurogenesis continues to occur throughout life but dramatically decreases with increasing age. This decrease is mostly related to a decline in proliferative activity as a result of an impoverishment of the microenvironment of the aged brain, including a reduction in trophic factors and increased inflammation.
\end{abstract}

Results: We determined that human umbilical cord blood mononuclear cells (UCBMC) given peripherally, by an intravenous injection, could rejuvenate the proliferative activity of the aged neural stem/progenitor cells. This increase in proliferation lasted for at least 15 days after the delivery of the UCBMC. Along with the increase in proliferation following UCBMC treatment, an increase in neurogenesis was also found in the aged animals. The increase in neurogenesis as a result of UCBMC treatment seemed to be due to a decrease in inflammation, as a decrease in the number of activated microglia was found and this decrease correlated with the increase in neurogenesis.

Conclusion: The results demonstrate that a single intravenous injection of UCBMC in aged rats can significantly improve the microenvironment of the aged hippocampus and rejuvenate the aged neural stem/progenitor cells. Our results raise the possibility of a peripherally administered cell therapy as an effective approach to improve the microenvironment of the aged brain.

\section{Background}

Aging is accompanied by a process of cellular senescence that occurs throughout the body, resulting in a decrease in the regenerative potential of the stem cell pools [1]. In the brain there are two stem cell pools, one residing in the subventricular zone (SVZ), and the other in the subgranular zone (SGZ) of the dentate gyrus of the hippocampus. As in other stem cell pools such as the hemapoietic pool 
in the bone marrow or the satellite stem cells in the muscle, the stem cells in the brain lose there capacity to generate new cells with age [2-4]. In the brain it appears that the decrease in neurogenesis is a result of a decrease in proliferation of the stem cells and not due to a loss of the cells [5]. In the muscle it has been shown that the stem cells can be rejuvenated by exposure of the cells to the systemic environment of a young animal through parabiosis [6]. Even though it has been known since the 1960s that a cellular senescence occurs with age [7], it is less clear if this cellular senescence leads to an aging phenotype, particularly to the age related cognitive decline.

However, it is clear that the process of cellular senescence that occurs with age is an important mechanism to protect against cancer. There are a number of tumor-supressor genes, including $\mathrm{p} 53$ and $\mathrm{p} 16^{\mathrm{ink} 4 \mathrm{~A}}$, which respond to cellular stressors to induce senescence [8]. It has recently been shown that knocking out p16 ink4A can restore the proliferative potential of the aged neural stem cells [9], but the animals have decreased longevity due to tumor formation [10]. This demonstrates the important balance that oncogenes play in protecting organisms from cancer, but with the negative consequence of inducing an aging state of cellular senescence. An effective target to lessen the amount of senescence might be the cellular stressors that accumulate with age which include telomere shorting [1], oxidative stress [11-13], inflammation [14], increased corticosteroid levels [15], and a decrease in a number of trophic factors including brain-derived neurotrophic factor (BDNF), vascular endothelial growth factor (VEGF), Insulin-like Growth Factor-1 (IGF-1) and fibroblast growth factor 2 (FGF-2) [16,17].

A potent cellular stressor that is increased with age is inflammation. Recently, our laboratory has shown that reducing neuroinflammation in aged rats by blocking the conversion of pro-IL- $1 \beta$ to IL- $1 \beta$ through inhibition of the converting enzyme caspase- 1 rescued some of the agerelated decrease in neurogenesis [18] and resulted in an improvement in cognitive function [19]. We believed that human umbilical cord blood mononuclear cells (UCBMC) may have a similar potential to restore some of the loss in capacity of the neural stem/progenitor cells ability to proliferate and differentiate into neurons.

In an animal model of stroke, UCBMC administered intravenously have reduced infarct volume and improved functional recovery on behavioral measures [20]. The effects of UCBMC have been attributed to changes in the microenvironment of the brain, through the release of trophic factors or by reducing inflammation, and not by a direct replacement of cells [21-23]. UCBMC contains a number of cell types including B-Cells and T-Cells, as well as, mesenchymal and endothelial progenitor cells.
UCBMC is also a rich source of $\mathrm{CD} 34^{+}$hematopoietic stem cells [24-26]. It was recently demonstrated that a systemic injection of UCBMC cells could suppress inflammation in the brain following stroke. Moreover, the effects of UCBMC cells seemed to shift the cytokine expression from a Th1 response to a Th2 response $[20,23,27]$. In addition to the immune modulatory effects, UCBMC cells also produce a number of trophic factors including, but not limited to, VEGF, nerve growth factor, and cytokine colony stimulating factor- 1 , thrombopoietin, and IL11 $[20,28,29]$.

The goal of the present study was to determine if UCBMC could stimulate the endogenous stem/progenitor cells to regenerate new cells. To this end, young and aged rats were intravenously administered a single dose of UCBMC to determine if UCBMC could increase proliferation of the neural stem/progenitor cells as well as to determine if there would be an effect on neurogenesis in the aged rats. This study provides insight into how the aged stem cell niche could be rejuvenated. Furthermore, as the UCBMC are administered minimally invasively this study raises the possibility of a clinically applicable therapeutic for the aged brain.

\section{Results \\ Human umbilical cord blood mononuclear cells (UCBMC) stimulate proliferation of the senescent hippocampal neural stem cell}

In the first of a series of experiments we wanted to determine if UCBMC given intravenously could stimulate the proliferation of the endogenous stem/progenitor cells in the hippocampus. We chose to study proliferation as a slowing of the cell cycle and a decrease in proliferation seems to be most affected with age when compared to the ability of the cells to survive and differentiate into neurons which appears to occur at relatively the same rate in young animals [30]. The effect of a single intravenous injection of UCBMC on cell proliferation in the granule cell layer in young (3-months old) or aged (20-months old) F344 rats was determined by analyzing BrdU staining 24 hours after the BrdU injections (48 hours following UCBMC injection). Using the optical fractionator method of design based stereology [31], we found that in the aged animals there was a significant increase $(\mathrm{t}(9)=4.256 ; \mathrm{p}<0.005)$ in the number of $\mathrm{BrdU}^{+}$cells in the UCBMC group (2504 $\pm 227.3 \mathrm{n}=5$ ) compared to the animals that received media alone $(1549 \pm 82.07 \mathrm{n}=6)$ (Figure $1 \mathrm{~A})$. In young animals there was no significant effect of the UCBMC treatment (data not shown). To determine if there might be a prolonged effect on proliferation in the aged F344 rats BrdU injections were given 14 days after the UCBMC treatment. Figure (1E) shows the effect of a single intravenous injection of UCBMC on the number of cells that incorporated BrdU on day 14. Stereological analysis 


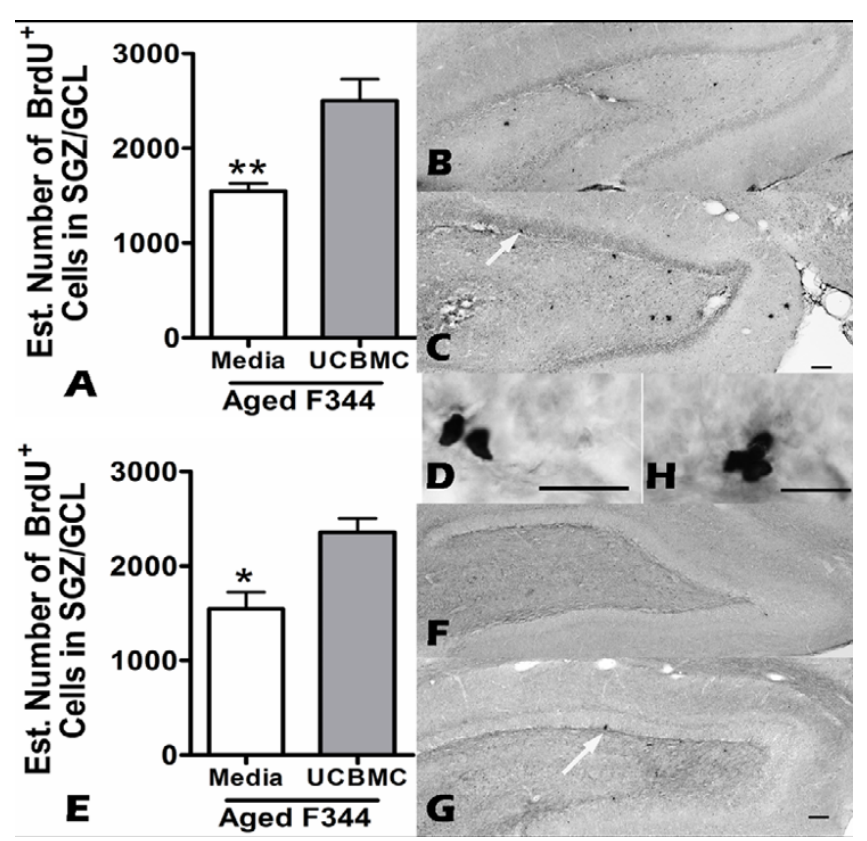

Figure I

Proliferation is increased in aged rats following

UCBMC treatment. To determine if UCBMC could stimulate proliferation of the hippocampal neural progenitor/ stem cells rats received two i.p. injections of BrdU (50 mg/ $\mathrm{kg}$ ) and were sacrifice the following day. (A) Quantification of the BrdU immunoreactive cell in the SGZ/GCL in aged rats 2 days after the UCBMC treatment showed that there was a significant $(P<0.005)$ increase in the number of BrdU immunoreactive cells. $(B, C)$ Photomicrographs of the dentate gyrus of a media-treated rat (B) and a UCBMC-treated rat (C) shows the BrdU staining in those animals sacrificed 2 days after the treatment. (D)The arrow in $C$ points to a cluster of BrdU immunoreactive cells from the UCBMC-treated rat shown in $D$ at higher magnification. $(E)$ To determine how long proliferation might remain elevated injections of $\mathrm{BrdU}(50 \mathrm{mg} / \mathrm{kg})$ began 14 days after the treatment. Quantification of the BrdU immunoreactive cells determine that the UCBMC-treated group had significantly $(p<0.01)$ more cells in the SGZ/GCL then the animals that received media alone. $(F, G) B r d U$ staining of the media-treated $(F)$ and the UCBMC-treated $(G)$ animals in the dentate gyrus of the hippocampus 15 days after the treatment. $(\mathrm{H})$ Arrow in $\mathrm{G}$ points to cells shown at higher magnification in $\mathrm{H}$. (scale bar for B, C, F, G is $100 \mu \mathrm{m}$; scale bar for $D, H$ is $25 \mu \mathrm{m}$ )

revealed that in the aged $U C B M C$-treated rats there was a significant increase in the number of BrdU+ cells $(\mathrm{t}(12)=$ 3.468; $\mathrm{p}<0.01)(2357 \pm 149.4 \mathrm{n}=6)$ compared to the media-treated group $(1548 \pm 176.9 \mathrm{n}=4)$.

\section{Neurogenesis is stimulated in the aged hippocampus following UCBMC treatment}

To determine if UCBMC would also stimulate neurogenesis in the aged rats, doublecortin (DCX) immunostaining was examined. Counting the number of $\mathrm{DCX}^{+}$cells in the SGZ/GCL, we found a significant increase $(\mathrm{t}(16)=2.188$; $\mathrm{p}<0.05)$ in the number of $\mathrm{DCX}^{+}$cells in the aged rats 15 days after a single i.v. injection of the UCBMC $(2619 \pm$ $212.6 \mathrm{n}=9)$ compared to animals that received media alone $(1843 \pm 283.9 \mathrm{n}=9)$ (Figure $2 \mathrm{~A})$. To confirm the results obtained by DCX, and to determine if there was any change in the ability of the proliferating cells to differentiate following a UCBMC treatment, we injected the animals with BrdU ( $50 \mathrm{mg} / \mathrm{kg}$ ) for five days beginning 24 hours after our i.v. treatment. Quantifying the number of $\mathrm{BrdU}^{+}$cells in the SGZ/GCL using the optical fractionator method of design based stereology we found a similar increase in the number of BrdU ${ }^{+}$cells in the aged UCBMC treated group as was found using the neurogenic marker DCX (Figure 2A-D). In aged rats, there was a significant increase $(F(2,14)=10.94, p<0.005)$ in the number of $\mathrm{BrdU}^{+}$cells generated over a period of five days following a single i.v. injection of UCBMC $(2772 \pm 263.3 \mathrm{n}=5)$ compared to the rats that received media alone (1498 \pm 206.1 $\mathrm{n}=5$ ); as determined by the Tukey's Multiple Comparison Test $(\mathrm{p}<0.01)$. In this experiment, we also included a group that was injected with adult human peripheral blood mononuclear cells (PBMC) as a control for the effect of delivering cells. The PBMC group was determined to have significantly fewer BrdU+ cells (1712 $\pm 171.2 \mathrm{n}=5)$ than the UCBMC treated group $(\mathrm{p}<0.01)$, but this was not significantly different from the group that received media alone (Figure $2 \mathrm{E}-\mathrm{H}$ ). As with the results of the proliferation study, young rats showed no significant effect of UCBMC treatment (data not shown). To determine if the treatment with UCBMC might alter the phenotype of the newborn cells, we double labeled with the antibodies to Tuj1, NeuN and GFAP. While exhaustive sampling was not conducted, $50 \mathrm{BrdU}^{+}$cells were analyzed from each rat (4 rats per group) using confocal microscopy for each marker and there did not appear to be any change in phenotype due to the treatment (Figure 2I-J). To confirm that the increase in neurogenesis was from the endogenous stem/progenitor cells, sections were stained for $\mathrm{HuNu}$ to look for the presence of the transplanted cells in the brain. Cells positive for the $\mathrm{HuNu}$ were found in the blood smears of the rats that were treated with UCBMC, but no HuNu immunoreactive cells were found in the hippocampus of the rats (data not shown).

\section{A decrease in microglia activation following UCBMC correlates with the increase in neurogenesis}

Using the optical fractionator method of design based stereology, we counted the number of OX-6+ cells in the dentate gyrus 15 days after a single UCBMC injection; this was at the same time point that we observed an increase in $\mathrm{DCX}^{+}$cells and BrdU ${ }^{+}$cells. OX-6 is a marker for MHCII and presumably stains for microglia in an activated, 


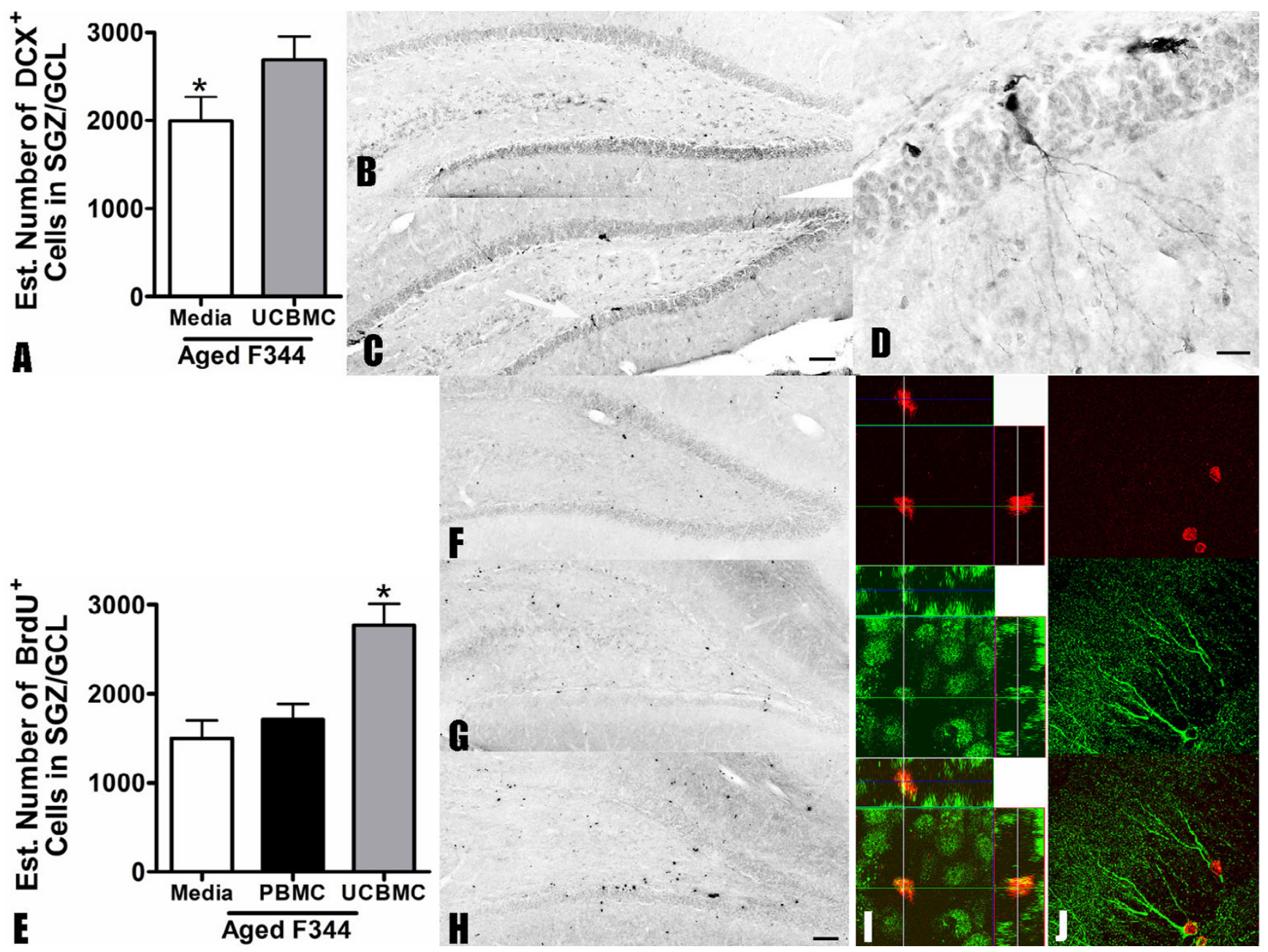

Figure 2

I 5 days after a UCBMC treatment neurogenesis is increase in aged rats. To determine if UCBMC treatment could stimulate neurogenesis aged $\mathrm{F} 344$ rats were sacrificed and immunohistochemical stained for DCX and $B r d U$. (A) $A$ significant increase $(p<0.05)$ in the number of $D_{C X}^{+}$cells, quantified in the SGZ/GCL, was found in the UCBMC treated rats. (B, C) Photomicrographs show the dentate gyrus demonstrating the DCX immunohistochemistry in the media-treated (B) and UCBMC-treated (C) rats. (D) A higher magnification photomicrograph of area indicated in $C$ shows a number of $D^{2} X^{+}$cells showing the different morphologies of the cells. (E) The results obtained with DCX were confirmed by BrdU. BrdU was injected i.p. for five consecutive days after the single injection of UCBMC. 10 days after the last injection of BrdU the animals were sacrificed. Compare to both a media control as well as an human adult peripheral blood (PBMC) control the UCBMC treated animals had significantly more $\mathrm{BrdU}^{+}$cells $(\mathrm{p}<0.0 \mathrm{I})$. ( $\left.\mathrm{F}, \mathrm{G}, \mathrm{H}\right)$ Photomicorgaphs of dentate gyrus shows BrdU immunohistochemistry in the media-treated $(F)$, PBMC-treated $(G)$ and UCBMC-treated $(H)$ rats. (I, J) Immunofluorescence was conducted to determine the phenotype of the BrdU cells. (I) An example of the cells double labeled with $\mathrm{BrdU}^{+} / \mathrm{NeuN}^{+}$(I; shown in orthogonal projection) and $\mathrm{BrdU}^{+} / \mathrm{TUJI}^{+}(\mathrm{J}$; shown using maximum projection). (scale bar for $\mathrm{B}, \mathrm{C}, \mathrm{F}, \mathrm{G}, \mathrm{H}$ is I00 $\mu \mathrm{m}$; scale bar for $\mathrm{D}$ is $25 \mu \mathrm{m}$ )

proinflammatory state. In aged rats, we found that 15 days after the UCBMC treatment there was a significant decrease $(\mathrm{t}(12)=2.699 ; \mathrm{p}<0.05)$ in the total number of activated OX-6+ microglia in the UCBMC group $(678.7 \pm$ $155.3 \mathrm{n}=7)$ compared to the media control (1217 \pm $128.0 \mathrm{n}=8$ ) (Figure $3 \mathrm{~A}$ ). The decrease in OX-6 $6^{+}$microglia negatively correlated with the number of $\mathrm{DCX}^{+}$cells (Spearman $r(15)=-0.6429 ; p<0.01)$ (Figure 3E).
Morphologically the OX- $6^{+}$cells expressed two main phenotypes (see Figure 3F). Type 1 microglia appear to be in a more quiescent state based on morphology Type 2 microglia were thought to represent a more activated state. The type 1 microglia make up the majority of the OX- $6^{+}$cells in the dentate gyrus and were found to be significantly decreased (unpaired $\mathrm{t}(12)=2.791 ; \mathrm{p}<0.05$ ) in aged rats following UCBMC treatment $(426.6 \pm 117.0 \mathrm{n}=$ 


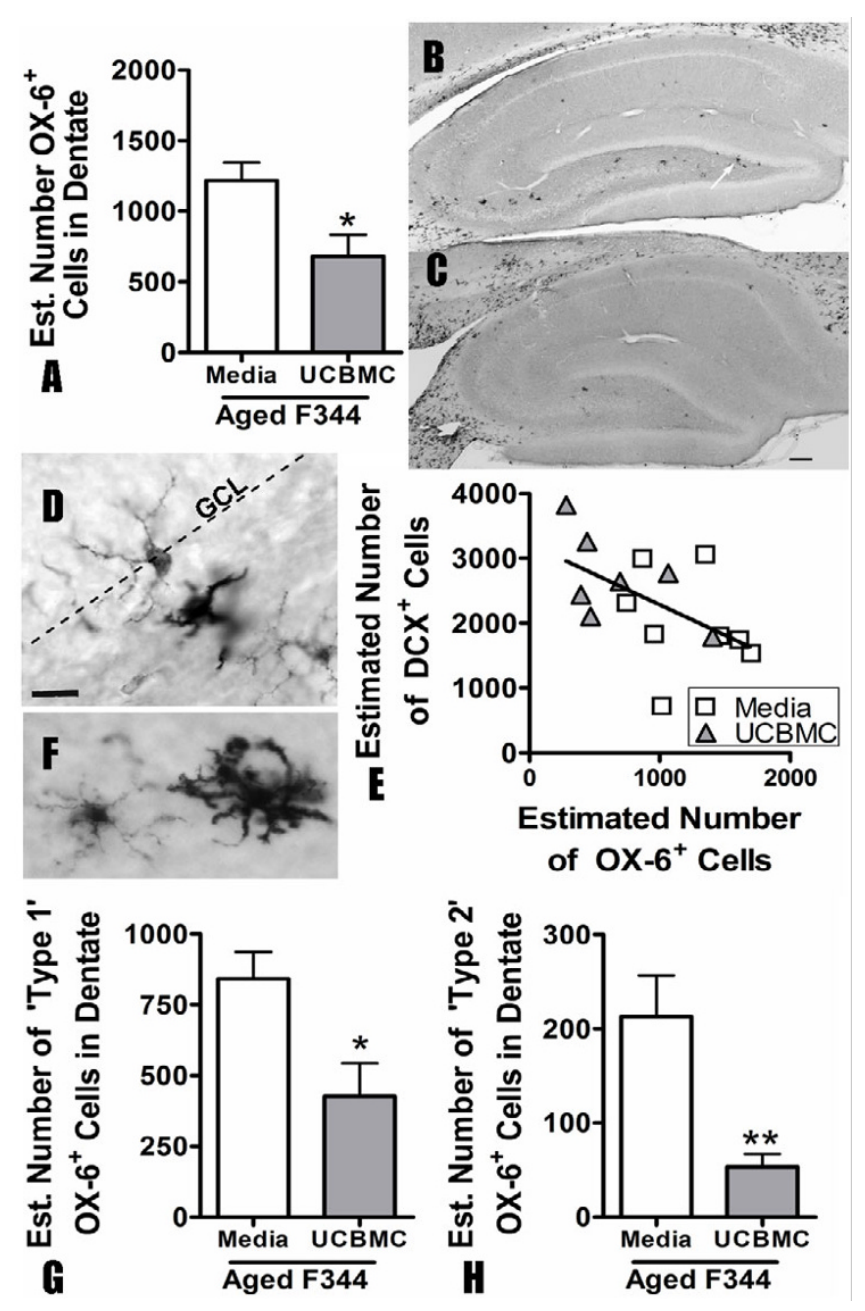

Figure 3

The decrease in microglia activation correlates with neurogenesis. I 5 days after the UCBMC treatment a significant reduction $(p<0.05)$ was found in the number of $O X-6^{+}$ cells in the dentate gyrus of the aged rats $(A)$. $(B, C)$ Photomicrographs are shown of the hippocampus of media-treated (C) and UCBMC-treated (C) rats. (D) A higher magnification photomicrograph of area indicated by arrow in B. (E) A significant negative correlation $(p<0.01)$ was found between the number of $O X-6^{+}$cells and the amount of neurogenesis as determine by the number of $\mathrm{DCX}^{+}$cells. (F) The OX-6 $6^{+}$ were further characterized based on morphology. The cell on the left represents a typical 'Type I' cell the cell on the right represents a typical 'Type 2' cell. Both 'Type I' $(p<$ $0.05 ; \mathrm{G})$ and 'Type 2 ' $(\mathrm{p}<0.0 \mathrm{I} ; \mathrm{H}) \mathrm{OX}-6^{+}$cells were significantly reduced in the aged animals following UCBMC treatment, but there was a greater reduction in 'Type 2' cells amounting to a four fold change. (scale bar for B, C is 200 $\mu \mathrm{m}$; scale bar for $\mathrm{D}$ is $25 \mu \mathrm{m}$ )

7) compared to controls $(842.4 \pm 94.67 \mathrm{n}=8)$ (Figure $3 \mathrm{G})$. The type 2 microglia, while representing a smaller percentage of the total OX-6+ microglia, were significantly reduced $(\mathrm{t}(12)=3.281 ; \mathrm{p}<0.01)$ to a greater extent by the UCBMC treatment $(53.30 \pm 13.27 \mathrm{n}=7)$ compared to controls $(212.9 \pm 43.82 \mathrm{n}=8)$ than the total microglia (Figure $3 \mathrm{H}$ ). Fifteen days after the UCBMC treatment, there was a 4 fold change in the number of type $2 \mathrm{OX}-6^{+}$ microglia, whereas there was only a 1.8 fold change in the total number of $\mathrm{OX}-6^{+}$microglia. It appears that the highly activated microglia are being reduced to a greater extent by the UCBMC treatment, although all OX- $6^{+}$ microglia are affected.

\section{Discussion}

The present study explored whether human umbilical cord blood mononuclear cells (UCBMC) could improve the neurogenic niche of the aged brain and stimulate the endogenous stem/progenitor cells to generate new neurons. As determined by stereological analysis of both DCX and BrdU, a single peripherally administered injection of UCBMC appeared to stimulate neurogenesis. The finding that the administration of UCBMC also increased the number of proliferative cells generated within 24 hours following the treatment, suggests that the increase in neurogenesis observed in this study may be a consequence of an increase in proliferation rather than changes in differentiation or survival of newly generated cells. To support this hypothesis, it will be important to allow more time for the cells to fully mature and then determine if there is still no change in the phenotype of the BrdU+ cells. It will also be important to determine what effect UCBMC have on the survival of the BrdU+ cells.

In addition, it was determined that UCBMC were able to increase cell proliferation for at least fifteen days in the aged rats. This suggests that the UCBMC may have a beneficial effect on the microenvironment of the aged brain. In support of this hypothesis we show that coinciding with an increase in neurogenesis in the aged treated rats, there was a decrease in the number of activated microglia in the dentate gyrus. A negative correlation between the degree of inflammation as indicated by the activation of microglia and the number of newborn neurons has been previously described [32]. Consistent with previous studies showing that UCBMC have the potential to reduce neuroinflammation $[20,23,27,30]$ in the aged brain, we did find that neurogenesis correlated with the number of activated microglia, suggesting that UCBMC were stimulating neurogenesis by decreasing microglia activation. Although other possibilities cannot be excluded, since UCBMC could be having multiple effects including increasing trophic support as previously published $[20,28,29]$.

UCBMC have been shown to reduce neuroinflammation $[20,23,27,30]$ and, consistent with previous studies, we show here that the peripherally administered UCBMC do 
have anti-inflammatory properties. It appears that one of the factors that leads to the negative regulation of neural stem cells is inflammation [32-34]. A primary source of inflammation in the CNS is from the macrophages/microglia which can produce a wide array of cytotoxic factors, including proinflammatory cytokines such as tumor necrosis factor (TNF), IL-1, IL-6 and IL-12 [35,36]. With age, microglia shift from a quiescent state into an active proinflammatory state. It is not clear if this change in activation state is in response to injury, infection, or debris or if it is due to dysregulated cytokine levels. Another possibility recently proposed, is that microglia becoming senescent and this leads to them becoming dysfunctional $[37,38]$. It has previously been demonstrated in models of induced inflammation through the use of LPS or radiation, a dramatic decrease in proliferation and neurogenesis occur, and when the inflammation is alleviated the replicative potential of the stem cells returns [32,34]. This effect is likely a protective mechanism so that the DNA is not exposed to the noxious inflammatory environment which could damage the replicating DNA. This correlation also imparts support to the hypothesis that UCBMC stimulate neurogenesis by decreasing inflammation, particularly the activation state of microglia. However, it does not rule out the possibility that UCBMC may be acting on multiple targets, with microglia only representing one part of the total mechanism.

While UCBMC do seem to have an effect on microglia, it is not clear how this occurs. A number of studies have shown that T-cells appear to act on macrophages/microglia to cause them to adopt a phenotype that is 'pro-repair' (i.e. the macrophages/microglia: clear debris, buffer toxic compounds, and produce growth factors), without being pro-inflammatory (i.e. producing TNF- $\alpha, \mathrm{NO}$, or COX-2) and this effect can promote neurogenesis and be neuroprotective [39-43]. As T-cells are a major fraction of $\mathrm{UCBMC}$, it is possible that the naïve T-cells in the UCBMC are able to induce a protective T-cell mediated response in the aged rats, since adult PBMC did not have an effect. Alternatively, the CD34+ stem cells in the UCBMC may be involved. Taguchi et al. [29] has shown that CD34+ stem cells can increase both angiogenesis and neurogenesis as part of the protective mechanism against stroke. From the results of the current study it can not be determined if the effects of the UCBMC are a result of direct action on the brain or though peripheral effect. However, the fact that we did not detect any immunoreactivity for human nuclei in the brains of the UCBMC-treated rats raises the possibility that the UCBMC may be acting through a peripheral mechanism. Moreover, the observation that the adult PBMC did not alter hippocampal neurogenesis ruled out the possibility of a non-specific effect due to an influx of cells, supporting our belief that the increase in neurogenesis, which occurred following treatment with UCBMC was not due to an influx of cells but was specific to UCBMC.

The present study did not attempt to determine if decreasing senescence of the neural stem cells could reverse the cognitive decline with age. There is still much debate surrounding the role of neurogenesis in learning and memory [44-49] and whether cellular senescence of the stem cell pool with age leads to an aging phenotype. While not a goal of the current study, it will be important to determine if the rejuvenation of the aged stem/progenitor cell pool can reverse the age-related cognitive decline.

In summary, this study demonstrates that a single peripheral injection of UCBMC could stimulate the endogenous neural stem/progenitor cells to increase proliferation. We also determined that the UCBMC were able to improve the microenvironment of the aged brain by reducing the number of activated microglia, and this reduction is correlated with an increase in neurogenesis. Further work will be important to determine the mechanism of action of UCBMC in the aged rats, including the possible role of the immune system in a T-cell mediated response, as well as the affects of angiogenesis via the $\mathrm{CD} 34^{+}$stem cells. It will also be important in future experiments to determine the duration that a single injection of UCBMC will elevated proliferation in aged rats. Not only do the results of this study provide novel insight into the state of the aged stem cell niche, the ability of the UCBMC to exert their effects while being administered minimally invasively may make translation to the clinical setting more likely. For this reason it will be important in future studies to determine the most efficacious dose and dosing regimen. Nevertheless, this is the first time that a systemic injection of hematopoietic cells has been shown to restore the regenerative potential of the aged brain, providing a novel insight into how the regenerative potential of the aged stem cell niches could be restored.

\section{Conclusion}

The results demonstrate that a single intravenous injection of UCBMC in aged rats can significantly improve the microenvironment of the aged hippocampus and rejuvenate the aged neural stem/progenitor cells. Our results raise the possibility of a peripherally administered cell therapy as an effective approach to improve the microenvironment of the aged brain.

\section{Methods \\ Cell preparation}

Cryopreserved human umbilical cord blood mononuclear cells (UCBMCs) were obtained from Saneron CCEL Therapeutics, Inc. (Tampa, FL, USA). Cryopreserved Human peripheral blood cells (PBMC) (mononuclear fraction) were obtained from AllCells, LLC (Emeryville, 
CA, USA). Just prior to intravenous (i.v.) injection, the UCBMC or PBMC were thawed into media (Hanks' balanced salt solution, HBSS, Gibco) at $37^{\circ} \mathrm{C}$, washed, and the number of viable cells was determined using the trypan blue exclusion method [23]. Cell viability ranged from 85 to $88 \%$. Cell concentration was adjusted to $10^{6}$ viable cells/500 $\mu \mathrm{l}$. Rats were then anesthetized with 3\% isofluorane and randomly chosen to receive a single i.v. injection via the penile vein of UCBMC at a dose $10^{6}$ cells shown most effective in a stroke model [22], 106 PBMC, or media for both the aged and young rats.

\section{Animals}

All experiments were conducted in accordance with the National Institute of Health Guide and Use of Laboratory Animals, and were approved by the Institutional Animal Care and Use committee of the University of South Florida, College of Medicine. Male Fisher 344 (F344) rats (NIA contract colony, Harlan Sprague Dawley, Indianapolis, IN), were pair-housed in environmentally controlled conditions $\left(12: 12 \mathrm{~h}\right.$ light:dark cycle at $\left.21 \pm 1^{\circ} \mathrm{C}\right)$ and provided food and water $a d$ lib. Two age groups of animals young ( 3 months old) and aged (20 months old) were used in this study. The mean life span of the F344 rats is approximately 29 months with a maximal life span of 36 months [50].

Rats were then divided in three groups. Group 1 received $50 \mathrm{mg} / \mathrm{kg}$ of bromodeoxyuridine (5-bromo-2-deoxyuridine, BrdU; Sigma, St. Louis, MO, USA), intraperitoneal (i.p.) twice a day beginning 24 hours the injection of UCBMC, and were sacrificed the subsequent day. Rats in group 2 received BrdU (50 mg/kg, i.p.) twice a day, beginning fourteen days after the administration of UCBMC and were sacrificed on the following day. Rats in Group 3 received $\mathrm{BrdU}$ ( $50 \mathrm{mg} / \mathrm{kg}$, i.p.) for five consecutive days, beginning the day after the administration of UCBMC and were the sacrificed day fifteen.

\section{Tissue collection and processing}

The rats were anesthetized with pentobarbital $(50 \mathrm{mg} / \mathrm{kg}$, i.p.). Blood was collected by cardiac puncture and smears were made of the blood to look for the presence of the transplanted cells. The rats were transcardiac perfusion with phosphate-buffered (PB), followed by $4 \%$ paraformaldehyde in PB. The brains were postfixed in $4 \%$ paraformaldehyde for $12 \mathrm{~h}$, after which they were transferred into 30\% sucrose in phosphate-buffered saline (PBS) for at least $16 \mathrm{~h}$, and stored at $4{ }^{\circ} \mathrm{C}$. Exhaustive sagittal sections of the left hemisphere were made, at $40 \mu \mathrm{m}$ using a Microm cryostat (Richard-Allan Scientific, Kalamazoo Michigan) and stored in cryoprotectant at $4{ }^{\circ} \mathrm{C}$.

\section{BrdU Immunohistochemistry}

All immunohistochemical staining was conducted on free-floating sections for every sixth section for the entire hippocampus beginning with a random start and including sections before and after the hippocampus to ensure that the entire structure was sampled; with one exception, in the aged animals from group 3 a one in three series was stained to allow for sampling of an adequate number of $\mathrm{BrdU}^{+}$cells. For BrdU staining, sections were pretreated with $50 \%$ formamide/ $2 \times$ SSC $(0.3 \mathrm{M} \mathrm{NaCl}, 0.03 \mathrm{M}$ sodium citrate) at $65^{\circ} \mathrm{C}$ for 2 hours, rinsed in $2 \times \mathrm{SSC}$, incubated in $2 \mathrm{~N} \mathrm{HCL}$ for 30 minutes at $37^{\circ} \mathrm{C}$, washed with borate buffer ( $\mathrm{pH} 8.5$ ), then PBS. This was followed by quenching endogenous peroxidase activity in $0.3 \%$ $\mathrm{H}_{2} \mathrm{O}_{2}$ solution in $30 \%$ methanol; then one hour in blocking solution (0.1 M PBS supplemented with 3\% normal horse serum and $0.25 \%$ Triton X-100: PBS-TS); followed by incubation overnight with mouse-anti-rat-BrdU (1:100; Roche) in PBS-TS. The following day the sections were washed and then incubated for one hour in a biotinylated secondary antibody (1:200; Vector Laboratories, Burlingame, CA) in PBS-TS; then washed before one hour incubation in avidin-biotin substrate (ABC kit, Vector Laboratories, Burlingame, CA); and then washed before 10 minutes incubation in DAB solution (Vector Laboratories, Burlingame, CA). Sections were then mounted onto glass slides and coverslipped with mounting medium.

\section{Doublecortin and OX-6 Immunohistochemistry}

Doublecortin (DCX) is a marker of migrating neurons that is expressed for approximately three weeks after the cell is born and has been shown to be a reliable indicator of neurogenesis $[51,52]$. For DCX immunohistochemistry a polyclonal goat antibody raised against human DCX (1:200; SC-8066, Santa Cruz biotechnology, Santa Cruz, CA, USA) was used following a similar protocol to BrdU except the antigen retrieval steps were omitted and Goat serum (Vector Laboratories, Burlingame, CA) was used instead of horse serum. For OX-6 immunohistochemistry a monoclonal antibody directed against the rat major histocompatibility II (MHCII) (RT1B, Becton, Dickinson Pharmingen, San Diego, CA, USA) was used at a concentration of 1:750 in place of the other primary antibodies all other steps were the same.

\section{Immunofluorescence}

Tissues were pretreated with 2 N HCL for 2 hours at room temperature, washed, and incubated in blocking solution (0.1 M PBS containing 10\% goat serum and $0.3 \%$ Triton $\mathrm{X}-100)$ for 1 hour at room temperature. Tissues were then incubated in rat anti-BrdU (1:400; Accurate Chemical, Westbury, NY) and additional primary antibodies [antiGFAP (1:500; Dako, Carpinteria, CA), mouse anti-NeuN (1:100; Chemicon, Temecula, CA), mouse anti-TUJ1 (1:800; Convance, Berkeley, CA)], overnight at $4^{\circ} \mathrm{C}$. Tis- 
sues were then rinsed 3 times in PBS and the appropriate secondary antibody conjugated to an Alexafluor probe (Molecular Probes, Eugene, OR) was applied for 2 hour. Following 6 washes in PBS, tissues were mounted on slides and coverslipped using Vectashield (Vector Labs, Burlingame, CA).

\section{Human Nuclei immunofluorescence}

To detect for the presence of the transplanted cells, blood smears and tissue sections were stained with a mouse monoclonal antibody that recognizes Human Nuclei antigen (HuNu) (MAB 1281; 1:50; Chemicon, Temecula, CA), and does not react with rat nuclei. Prior to incubation overnight at $4^{\circ} \mathrm{C}$ in the $\mathrm{HuNu}$ antibody, the samples were washed in PBS and incubated in blocking solution (0.1 M PBS containing $10 \%$ goat serum and $0.3 \%$ Triton $\mathrm{X}-100)$ for 1 hour at room temperature. The HuNu antibody was visualized by secondary antibody conjugated to an Alexafluor probe (Molecular Probes, Eugene, OR).

\section{Quantification and imaging}

To determine cell numbers the optical fractionator method of unbiased stereological cell counting techniques [31] was used with a Nikon Eclipse 600 microscope and quantified using Stereo Investigator software (MicroBrightField, Colchester, VT). For the proliferation study, because of the low number of $\mathrm{BrdU}^{+}$cell in the aged animals the virtual grid and counting frame were both $125 \mu \mathrm{m} \times 125 \mu \mathrm{m}$ in order to count all the cells that were present in the section. For all other counts sampling was optimized to count at least 200 cells per animal with error coefficients less than 0.07 . Outlines of the anatomical structures were done using a $10 \times / 0.45$ objective and cell quantification was conducted using a $60 \times / 1.40$ objective. OX-6+ $6^{+}$cells were counted in the entire dentate gyrus including the subgranular zone (SGZ: defined as a two cell diameter band on both sides of the granular cell layer (GCL)). All other cell counts were done in the SGZ/GCL. The phenotype of the $\mathrm{BrdU}^{+}$cells were analyzed using an inverted Zeiss LSM 510 confocal microscope with a 40×/ 1.30 NA oil immersion objective. Argon and HeNe laser lines in conjunction with 488 and 555 band pass filters were applied to excite the samples using line switching to minimize crosstalk between fluorochromes. Images and Z-stacks were produced with dual photomultiplier detectors and the LSM 5 version 3,2,0,115 software suite, and optical $\mathrm{Z}$ stacks where created at $2 \mu \mathrm{m}$ intervals throughout the $40 \mu \mathrm{m}$ of the sections with a guard region of $2 \mu \mathrm{m}$ excluded from top and bottom of the $\mathrm{Z}$ stack. The $\mathrm{Z}$ stacks were rotated in all planes to verify double labeling.

\section{Statistical analyses}

Data are presented as mean cell number \pm SEM. Statistical analysis was performed using an unpaired, two-side $t$-test, or a one-way ANOVA followed by a Tukeys post-hoc test. $p$ $<0.05$ was considered to be significant.

\section{Abbreviations}

GCL (granular cell layer), PBMC (peripheral blood mononuclear cells), UCBMC (umbilical cord blood mononuclear cells), SGZ (subgranular zone)

\section{Competing interests}

PCB, AEW are consultants to Saneron CCEL Therapeutics Inc (SCTI). PRS is a co-founder of SCTI. AEW \& PRS are inventors of UCBMC related patents applications.

\section{Authors' contributions}

ADB, PRS, AEW, PCB, CG designed research. ADB, MMP, MJC, CEH, CG performed research. ADB wrote paper. All authors read and approved the final manuscript.

\section{Acknowledgements}

This work was supported by: NIH grant R2IAG024I65 (CG), POIAG044I 8 (PCB), and R0IAG020927(AEW); The US Veterans Administration Medical Research Service; In part by, the Analytic Microscopy Core Facility at the H. Lee Moffitt Cancer Center and Research Institute. The UCBMC were generously donated by Saneron CCEL Therapeutics Inc. Thanks are due to Ning Chen, and Craig T. Ajmo Jr., for their technical assistance.

\section{References}

I. Collado M, Blasco MA, Serrano M: Cellular senescence in cancer and aging. Cell 2007, I 30(2):223-233.

2. Cameron HA, McKay RDG: Restoring production of hippocampal neurons in old age. Nat Neurosci 1999, 2(10):894-897.

3. Kronenberg G, Bick-Sander A, Bunk E, Wolf C, Ehninger D, Kempermann G: Physical exercise prevents age-related decline in precursor cell activity in the mouse dentate gyrus. Neurobiology of Aging 2006, 27(10): I 505-1513.

4. Kuhn HG, Dickinson-Anson H, Gage FH: Neurogenesis in the dentate gyrus of the adult rat: age-related decrease of neuronal progenitor proliferation. I Neurosci 1996, 16(6):2027-2033.

5. Hattiangady B, Shetty AK: Aging does not alter the number or phenotype of putative stem/progenitor cells in the neurogenic region of the hippocampus. Neurobiol Aging 2006.

6. Conboy IM, Conboy MJ, Wagers AJ, Girma ER, Weissman IL, Rando TA: Rejuvenation of aged progenitor cells by exposure to a young systemic environment. Nature 2005, 433(7027):760-764.

7. Hayflick L, Moorhead PS: The serial cultivation of human diploid cell strains. Exp Cell Res 1961, 25:585-62I.

8. Campisi J: Senescent cells, tumor suppression, and organismal aging: good citizens, bad neighbors. Cell 2005, I 20(4):5। 3-522.

9. Molofsky AV, Slutsky SG, Joseph NM, He S, Pardal R, Krishnamurthy J, Sharpless NE, Morrison SJ: Increasing pI6INK4a expression decreases forebrain progenitors and neurogenesis during ageing. Nature 2006, 443(7 I I 0):448-452.

10. Beausejour CM, Campisi J: Ageing: balancing regeneration and cancer. Nature 2006, 443(7 I I 0):404-405.

1I. Ames BN, Shigenaga MK: Oxidants are a major contributor to aging. Annals of the New York Academy of Sciences 1992, 663:85-96.

12. Ames BN, Shigenaga MK, Hagen TM: Oxidants, antioxidants, and the degenerative diseases of aging. Proceedings of the National Academy of Sciences of the United States of America 1993, 90( I 7):7915-7922.

13. Harman D: Aging: a theory based on free radical and radiation chemistry. Journal of gerontology 1956, I I (3):298-300.

14. Blalock EM, Chen KC, Sharrow K, Herman JP, Porter NM, Foster TC, Landfield PW: Gene Microarrays in Hippocampal Aging: Sta- 
tistical Profiling Identifies Novel Processes Correlated with Cognitive Impairment. 2003, 23(9):3807-3819.

15. Sapolsky RM: Do glucocorticoid concentrations rise with age in the rat? Neurobiol Aging 1992, I3(I): I7I-I74.

16. Hattiangady B, Rao MS, Shetty GA, Shetty AK: Brain-derived neurotrophic factor, phosphorylated cyclic AMP response element binding protein and neuropeptide $Y$ decline as early as middle age in the dentate gyrus and CAI and CA3 subfields of the hippocampus. Experimental Neurology 2005, 195(2):353-371.

17. Shetty AK, Hattiangady B, Shetty GA: Stem/progenitor cell proliferation factors FGF-2, IGF-I, and VEGF exhibit early decline during the course of aging in the hippocampus: role of astrocytes. Glia 2005, 5 I(3): 173-186.

18. Gemma C, Bachstetter AD, Cole M, Fister M, Hudson C, Bickford PC: Blockade of Caspase-I Increases Neurogenesis in the Aged Hippocampus. The European journal of neuroscience 2007 in press.

19. Gemma C, Fister M, Hudson C, Bickford PC: Improvement of memory for context by inhibition of caspase- $\mathrm{I}$ in aged rats. The European journal of neuroscience 2005, 22(7): I75I-1756.

20. Vendrame M, Cassady J, Newcomb J, Butler T, Pennypacker KR, Zigova T, Sanberg CD, Sanberg PR, Willing AE: Infusion of human umbilical cord blood cells in a rat model of stroke dosedependently rescues behavioral deficits and reduces infarct volume. Stroke 2004, 35( I 0):2390-2395.

21. Borlongan CV, Hadman M, Sanberg CD, Sanberg PR: Central nervous system entry of peripherally injected umbilical cord blood cells is not required for neuroprotection in stroke. Stroke 2004, 35(10):2385-2389.

22. Newman MB, Willing AE, Manresa J], Sanberg CD, Sanberg PR: Cytokines produced by cultured human umbilical cord blood (HUCB) cells: Implications for brain repair. Experimental Neurology 2006, I 99(I):20I-208.

23. Vendrame M, Gemma C, de Mesquita D, Collier L, Bickford PC, Sanberg CD, Sanberg PR, Pennypacker KR, Willing AE: Anti-inflammatory effects of human cord blood cells in a rat model of stroke. Stem Cells Dev 2005, I 4(5):595-604.

24. Bender JG, Unverzagt KL, Walker DE, Lee W, Van Epps DE, Smith $\mathrm{DH}$, Stewart CC, To LB: Identification and comparison of CD34-positive cells and their subpopulations from normal peripheral blood and bone marrow using multicolor flow cytometry. Blood I99I, 77( I 2):259I-2596.

25. Ho AD, Young D, Maruyama M, Corringham RE, Mason JR, Thompson P, Grenier K, Law P, Terstappen LW, Lane T: Pluripotent and lineage-committed CD34+ subsets in leukapheresis products mobilized by G-CSF, GM-CSF vs. a combination of both. Experimental hematology 1996, 24(13): |460-| 468.

26. Wu AG, Michejda M, Mazumder A, Meehan KR, Menendez FA, Tchabo JG, Slack R, Johnson MP, Bellanti JA: Analysis and characterization of hematopoietic progenitor cells from fetal bone marrow, adult bone marrow, peripheral blood, and cord blood. Pediatric research 1999, 46(2): 163-169.

27. Vendrame M, Gemma C, Pennypacker KR, Bickford PC, Davis Sanberg C, Sanberg PR, Willing AE: Cord blood rescues strokeinduced changes in splenocyte phenotype and function. Experimental Neurology 2006, 199(I): I91-200.

28. Suen Y, Lee SM, Schreurs J, Knoppel E, Cairo MS: Decreased macrophage colony-stimulating factor mRNA expression from activated cord versus adult mononuclear cells: altered posttranscriptional stability. Blood 1994, 84(1 2):4269-4277.

29. Taguchi A, Soma T, Tanaka H, Kanda T, Nishimura $H$, Yoshikawa $H$, Tsukamoto $Y$, Iso H, Fujimori Y, Stern DM, Naritomi H, Matsuyama T: Administration of CD34+ cells after stroke enhances neurogenesis via angiogenesis in a mouse model. J Clin Invest 2004, I I 4(3):330-338.

30. Rao MS, Hattiangady B, Abdel-Rahman A, Stanley DP, Shetty AK: Newly born cells in the ageing dentate gyrus display normal migration, survival and neuronal fate choice but endure retarded early maturation. The European journal of neuroscience 2005, 2 I (2):464-476.

31. West MJ, Slomianka L, Gundersen HJ: Unbiased stereological estimation of the total number of neurons in thesubdivisions of the rat hippocampus using the optical fractionator. The Anatomical record I991, 23 I(4):482-497.
32. Ekdahl CT, Claasen JH, Bonde S, Kokaia Z, Lindvall O: Inflammation is detrimental for neurogenesis in adult brain. Proc Natl Acad Sci U S A 2003, I00(23):13632-13637.

33. Battista D, Ferrari CC, Gage FH, Pitossi FJ: Neurogenic niche modulation by activated microglia: transforming growth factor beta increases neurogenesis in the adult dentate gyrus. The European journal of neuroscience 2006, 23(I):83-93.

34. Monje ML, Toda H, Palmer TD: Inflammatory blockade restores adult hippocampal neurogenesis. Science 2003 , 302(565I): 1760-1765.

35. Gao HM, Jiang J, Wilson B, Zhang W, Hong JS, Liu B: Microglial activation-mediated delayed and progressive degeneration of rat nigral dopaminergic neurons: relevance to Parkinson's disease. Journal of neurochemistry 2002, 8 I (6): I 285- 1297.

36. Mantovani A, Sica A, Sozzani S, Allavena P, Vecchi A, Locati M: The chemokine system in diverse forms of macrophage activation and polarization. Trends in immunology 2004, 25(I 2):677-686.

37. Schwartz M, Butovsky O, Bruck W, Hanisch UK: Microglial phenotype: is the commitment reversible? Trends in Neurosciences 2006, 29(2):68-74.

38. Streit W]: Microglial senescence: does the brain's immune system have an expiration date? Trends in Neurosciences 2006, 29(9):506-5। 0.

39. Butovsky O, Ziv Y, Schwartz A, Landa G, Talpalar AE, Pluchino S, Martino G, Schwartz M: Microglia activated by IL-4 or IFN[gamma] differentially induce neurogenesis and oligodendrogenesis from adult stem/progenitor cells. Molecular and Cellular Neuroscience 2006, 3 I ( I): | 49-160.

40. Shaked I, Porat Z, Gersner R, Kipnis J, Schwartz M: Early activation of microglia as antigen-presenting cells correlates with $T$ cell-mediated protection and repair of the injured central nervous system. J Neuroimmunol 2004, I 46(I-2):84-93.

4I. Ziv Y, Avidan H, Pluchino S, Martino G, Schwartz M: Synergy between immune cells and adult neural stem/progenitor cells promotes functional recovery from spinal cord injury. Proc Natl Acad Sci U S A 2006, 1 03(35): I 3 | 74 - 13179.

42. Ziv Y, Finkelstein A, Geffen Y, Kipnis J, Smirnov I, Shpilman S, Vertkin I, Kimron M, Lange A, Hecht T, Reyman KG, Marder JB, Schwartz M, Yoles $E$ : A novel immune-based therapy for stroke induces neuroprotection and supports neurogenesis. Stroke 2007, 38(2 Suppl):774-782.

43. Ziv Y, Ron N, Butovsky O, Landa G, Sudai E, Greenberg N, Cohen H, Kipnis J, Schwartz M: Immune cells contribute to the maintenance of neurogenesis and spatial learning abilities in adulthood. Nat Neurosci 2006, 9(2):268-275

44. Gould E, Tanapat P, Hastings NB, Shors TJ: Neurogenesis in adulthood: a possible role in learning. Trends Cogn Sci 1999 , 3(5): $186-192$

45. Kee N, Teixeira CM, Wang AH, Frankland PW: Preferential incorporation of adult-generated granule cells into spatial memory networks in the dentate gyrus. Nat Neurosci 2007, I0(3):355-362.

46. Leuner B, Gould E, Shors T]: Is there a link between adult neurogenesis and learning? Hippocampus 2006, 16(3):216-224.

47. Merrill DA, Karim R, Darraq M, Chiba AA, Tuszynski MH: Hippocampal cell genesis does not correlate with spatial learning ability in aged rats. J Comp Neurol 2003, 459(2):20I-207.

48. Shors TJ, Miesegaes G, Beylin A, Zhao M, Rydel T, Gould E: Neurogenesis in the adult is involved in the formation of trace memories. Nature 200I, 4I0(6826):372-376.

49. Shors T], Townsend DA, Zhao M, Kozorovitskiy Y, Gould E: Neurogenesis may relate to some but not all types of hippocampaldependent learning. Hippocampus 2002, I 2(5):578-584

50. Coleman GL, Barthold W, Osbaldiston GW, Foster SJ, Jonas AM Pathological changes during aging in barrier-reared Fischer 344 male rats. J Gerontol 1977, 32(3):258-278.

5I. Couillard-Despres S, Winner B, Schaubeck S, Aigner R, Vroemen M, Weidner N, Bogdahn U, Winkler J, Kuhn HG, Aigner L: Doublecortin expression levels in adult brain reflect neurogenesis. The European journal of neuroscience 2005, 2 I (I): I-I4.

52. Rao MS, Shetty AK: Efficacy of doublecortin as a marker to analyse the absolute number and dendritic growth of newly generated neurons in the adult dentate gyrus. The European journal of neuroscience 2004, 19(2):234-246. 